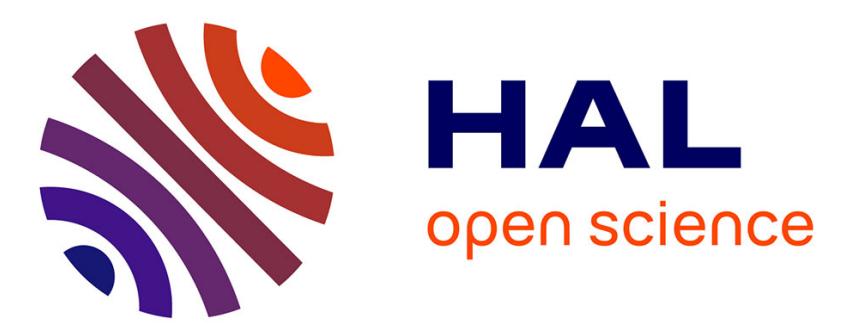

\title{
Array synthesis problems via convex relaxation
}

Benjamin Fuchs

\section{To cite this version:}

Benjamin Fuchs. Array synthesis problems via convex relaxation. APSURSI 2014: Antennas and Propagation Society International Symposium, 2014, pp.1361 - 1362. 10.1109/APS.2014.6905006 . hal-01082699

\section{HAL Id: hal-01082699 https://hal-univ-rennes1.archives-ouvertes.fr/hal-01082699}

Submitted on 14 Nov 2014

HAL is a multi-disciplinary open access archive for the deposit and dissemination of scientific research documents, whether they are published or not. The documents may come from teaching and research institutions in France or abroad, or from public or private research centers.
L'archive ouverte pluridisciplinaire HAL, est destinée au dépôt et à la diffusion de documents scientifiques de niveau recherche, publiés ou non, émanant des établissements d'enseignement et de recherche français ou étrangers, des laboratoires publics ou privés. 


\title{
Array Synthesis Problems via Convex Relaxation
}

\author{
Benjamin Fuchs \\ University of Rennes 1, IETR \\ Rennes, France \\ benjamin.fuchs@univ-rennes1.fr
}

\begin{abstract}
A general procedure, based on the SemiDefinite Relaxation (SDR) technique, is presented to solve efficiently a wide range of difficult (because non-convex) array synthesis problems. This powerful approximation technique is easy to implement and the so-approximated problem can then be efficiently solved using off-the-shelf numerical routines. Examples of shaped beam and reconfigurable array synthesis are shown to illustrate the potentialities of the proposed approach.
\end{abstract}

\section{INTRODUCTION}

A large number of array synthesis problems (such as the synthesis of shaped beams or phase-only arrays for instance) are difficult optimization problems because of their non convexity. In this paper, a general procedure based on the SemiDefinite Relaxation (SDR) technique is proposed to approximate in order to solve efficiently such difficult array synthesis problems. The SDR technique has lately shown a great significance and relevance on many applications in signal processing and communications as reviewed in [1]. It has been recently applied successfully to synthesize phased arrays with notches in the beam pattern [2], [3].

The main principle of the SDR and the way to apply this powerful and computationally efficient approximation technique to the synthesis of various antenna array are here detailed.

\section{Problem Formulation and Resolution}

The far field $f(\theta)$ radiated in the direction $\theta$ by an antenna array composed of $N$ elements is the product between the steering vector $\boldsymbol{a}(\theta)$ and the complex (magnitude and phase) excitation vector $\boldsymbol{w}$ :

$$
f(\theta)=\boldsymbol{a}(\theta)^{H} \boldsymbol{w},
$$

where ${ }^{H}$ denotes the Hermitian transposition. Let us introduce the notation $f_{i}=f\left(\theta_{i}\right)$ and $\boldsymbol{a}_{i}=\boldsymbol{a}\left(\theta_{i}\right)$. The real value version of (1) in the direction $\theta_{i}$ is then:

$$
\begin{aligned}
& {\left[\mathbb{R}\left(f_{i}\right) \mathbb{I}\left(f_{i}\right)\right]^{T}=\boldsymbol{A}_{i} \boldsymbol{x},} \\
& \text { with } \boldsymbol{A}_{i}=\left[\begin{array}{rr}
\mathbb{R}\left(\boldsymbol{a}_{i}{ }^{T}\right) & -\mathbb{I}\left(\boldsymbol{a}_{i}^{T}\right) \\
\mathbb{I}\left(\boldsymbol{a}_{i}{ }^{T}\right) & \mathbb{R}\left(\boldsymbol{a}_{i}^{T}\right)
\end{array}\right] \text { and } \boldsymbol{x}=\left[\begin{array}{l}
\mathbb{R}(\boldsymbol{w}) \\
\mathbb{I}(\boldsymbol{w})
\end{array}\right]
\end{aligned}
$$

where $\boldsymbol{A}_{i} \in \mathbb{R}^{2 \times 2 N}, \boldsymbol{x} \in \mathbb{R}^{2 N \times 1}, .^{T}$ is the transpose operator, $\mathbb{R}$ and $\mathbb{I}$ stands for the real and imaginary parts respectively. The power radiated by the array is then:

$$
\left|f_{i}\right|^{2}=\boldsymbol{x}^{T} \boldsymbol{Q}_{i} \boldsymbol{x}, \text { with } \boldsymbol{Q}_{i}=\boldsymbol{A}_{i}^{T} \boldsymbol{A}_{i} .
$$

Note that in the formulation (3), the power radiated by arbitrary arrays, i.e. arrays of any given geometry and composed of elements with any known radiation patterns, can be considered.
Let us recall that for any real symmetric matrix $\boldsymbol{C}$ and any real vector $\boldsymbol{x}$ :

$$
\boldsymbol{x}^{T} \boldsymbol{C} \boldsymbol{x}=\operatorname{Tr}\left(\boldsymbol{x}^{T} \boldsymbol{C} \boldsymbol{x}\right)=\operatorname{Tr}\left(\boldsymbol{C} \boldsymbol{x} \boldsymbol{x}^{T}\right) .
$$

Using (4), the power (3) radiated by the array becomes:

$$
\left|f_{i}\right|^{2}=\operatorname{Tr}\left(\boldsymbol{Q}_{i} \boldsymbol{X}\right) \text {, with } \boldsymbol{X}=\boldsymbol{x} \boldsymbol{x}^{T} \in \mathbb{R}^{2 N \times 2 N} \text {. }
$$

At this step, it is important to observe that $\boldsymbol{X}=\boldsymbol{x} \boldsymbol{x}^{T}$ is equivalent to $\boldsymbol{X}$ being a symmetric positive semidefinite matrix (denoted $\boldsymbol{X} \succeq 0)$ of rank one $(\operatorname{rank}(\boldsymbol{X})=1)$.

Let us now consider a typical array synthesis problem in order to explain the SDR technique. Many synthesis problems amount to look for the array excitations $\boldsymbol{x}$ such that the power radiated by the array is constrained or equivalently $\left|f_{i}\right|^{2}$ belongs to a set $\mathcal{C}_{i}$ for the directions $i=1, \ldots, I$ :

$$
\text { find } \boldsymbol{x} \text { such that }\left|f_{i}\right|^{2} \in \mathcal{C}_{i}= \begin{cases}\left|f_{i}\right|^{2} \leq u_{i}, & i=1, \ldots, I_{1} \\ \left|f_{i}\right|^{2} \geq l_{i}, & i=1, \ldots, I_{2} \\ \left|f_{i}\right|^{2}=d_{i}, & i=1, \ldots, I_{3}\end{cases}
$$

With (4) and (5), the problem (6) is equivalent to:

$$
\text { find } \boldsymbol{X} \text { such that }\left\{\begin{array}{l}
\operatorname{Tr}\left(\boldsymbol{Q}_{i} \boldsymbol{X}\right) \in \mathcal{C}_{i}, \text { for } i=1, \ldots, I \\
\boldsymbol{X} \succeq 0 \text { and } \operatorname{rank}(\boldsymbol{X})=1
\end{array}\right. \text {. }
$$

By dropping the non-convex rank constraint, we obtain an approximation of (7) that is called SemiDefinite Relaxation (SDR) since it is an instance of semidefinite programming. The SDR formulation is convenient because it can be solved optimally by readily available software such as CVX [4].

The main issue is then to transform the globally optimal solution $\boldsymbol{X}^{*}$ of the SDR into a feasible point $\tilde{\boldsymbol{x}}$ of the original synthesis problem (6). If $\operatorname{rank}\left(\boldsymbol{X}^{*}\right)=1$, then $\boldsymbol{X}^{*}=\boldsymbol{x}^{*} \boldsymbol{x}^{* T}$ and $\boldsymbol{x}^{*}$ is not only a feasible point but also the optimal solution of (6). In general, the solution $\boldsymbol{X}^{*}$ of the SDR is such as $\operatorname{rank}\left(\boldsymbol{X}^{*}\right)>1$. In that case, we take the best rank-one approximation (in the least two norm sense) $\boldsymbol{X}_{1}^{*}$ of $\boldsymbol{X}^{*}$. It can be obtained via an eigenvalue decomposition [6] as follows:

$$
\boldsymbol{X}_{1}^{*}=\sigma_{1} \boldsymbol{u}_{1} \boldsymbol{u}_{1}^{T},
$$

where $\sigma_{1}$ is the largest eigenvalue of $\boldsymbol{X}^{*}$ and $\boldsymbol{u}_{1}$ is the corresponding eigenvector. The vector $\tilde{\boldsymbol{x}}=\sqrt{\sigma_{1}} u_{1}$ is then a potential solution of (6) provided that it is a feasible solution. Note that there are many efficient techniques to foster lowrank solutions as reviewed in [5] and thereby induce that the 
solution $\boldsymbol{X}_{1}^{*}$ is very close to $\boldsymbol{X}^{*}$.

Finally, it is important to point out that even though the extracted solution $\tilde{\boldsymbol{x}}$ is feasible for (6), there is no guarantee that it is an optimal solution. For otherwise, it would mean that we have solved a NP-hard problem in a polynomial time.

\section{NUMERICAL APPLICATIONS}

\section{A. Shaped Beam Synthesis}

The synthesis of a cosecant beam with a linear array composed of 30 isotropic elements that are half wavelength spaced is considered. An heuristic procedure (tabu search algorithm) has been used to find the array excitations in [7]. The proposed approach also manages to find a solution that satisfies this stringent far field template. The far field patterns and array element excitations are plotted in Fig. 1.
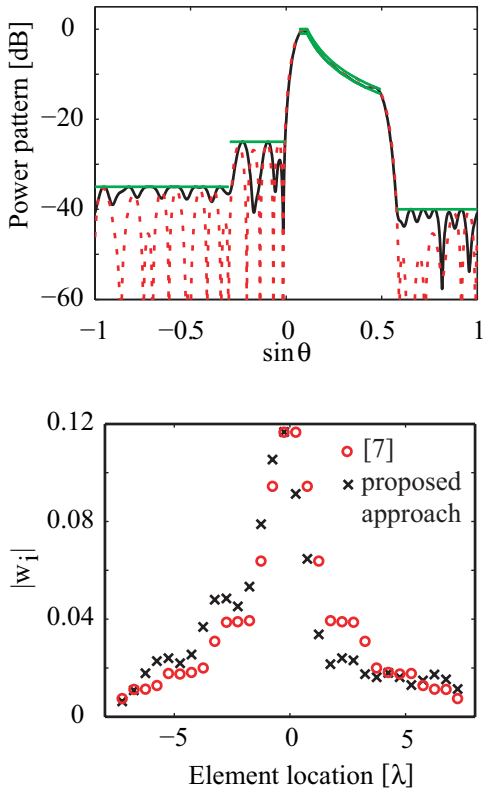

Fig. 1. Cosecant beam synthesis of a 30 element linear array.

\section{B. Synthesis of Reconfigurable Array by Phase-Only Control}

We consider an example of reconfigurable array synthesis that is presented in [8]. The goal is to determine the complex excitations of a linear equispaced array composed of 20 half wavelength spaced isotropic elements. By only changing the phases of the excitations, the pattern radiated by the array must switch from a focused to a shaped sectoral beam and vice versa.

The synthesized far field patterns and array element excitations are plotted in Fig. 2. The proposed approach allows to determine at once (in less than $30 \mathrm{~s}$ on a standard laptop) both the common excitation magnitudes and different phases to generate the focused and shaped beam patterns.

More details about how to apply the SDR to array synthesis problems and additional applications examples can be found in [9].
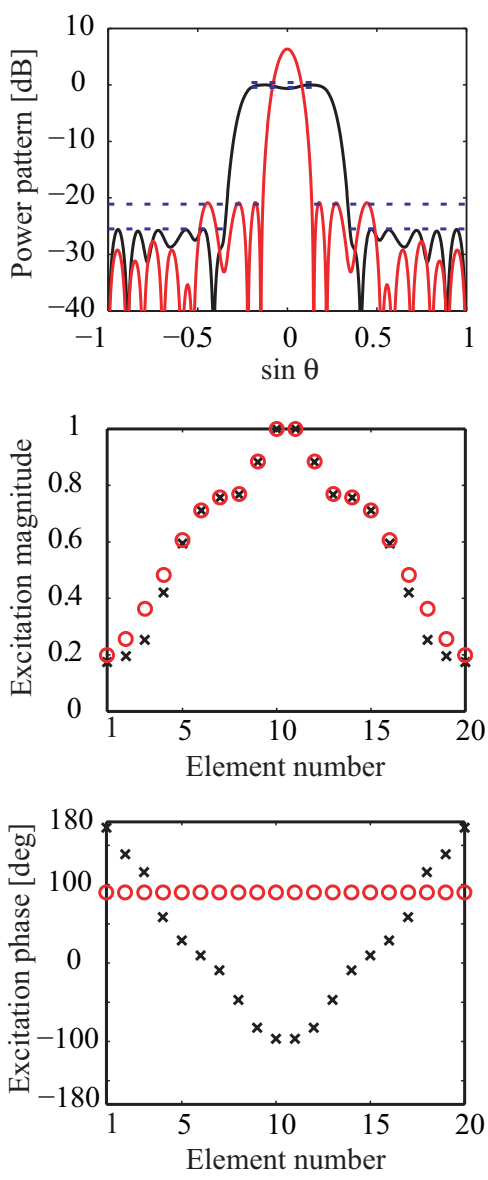

Fig. 2. Synthesis results of reconfigurable array by phase only control.

\section{REFERENCES}

[1] Z.-Q. Luo, W.-K. Ma, A. Man-Cho So, Y. Ye, and S. Zhang, "Semidefinite Relaxation of Quadratic Optimization Problems," IEEE Signal Processing Magazine, vol. 27, no. 3, pp. 20-34, May 2010.

[2] P.J. Kajenski, "Phase Only Antenna Pattern Notching Via a Semidefinite Programming Relaxation," IEEE Trans. on Antennas and Propagation, vol. 60, no. 5, pp. 2562-2565, May 2012.

[3] P.J. Kajenski, "Phase-Only Monopulse Pattern Notching Via a Semidefinite Programming," APS, Chicago, 2012.

[4] CVX Research, Inc. CVX: Matlab software for disciplined convex programming, version 2.0 beta. http://cvxr.com/cvx, September 2012.

[5] M. Fazel, H. Hindi, and S. Boyd, "Rank Minimization and Applications in System Theory," Proc. American Control Conference, Boston, Massachusetts, June 2004.

[6] G. Strang, "Introduction to Linear Algebra," Wellesley Cambridge Press, 4th edition, 2009.

[7] A. Akdagli and K. Guney, "Shaped-beam pattern synthesis of equally and unequally spaced linear antenna arrays using a modified tabu search algorithm," Microwave Opt. Technol. Lett., vol. 36, no. 1, pp. 16-20, Jan. 2003.

[8] A.F. Morabito, A. Massa, P. Rocca, and T. Isernia, "An Effective Approach to the Synthesis of Phase-Only Reconfigurable Linear Array," IEEE Trans. on Antennas and Propagation, vol. 60, no. 8, pp. 3622-3631, Aug. 2012.

[9] B. Fuchs, "Application of Convex Relaxation to Array Synthesis Problems," accepted for publication in IEEE Trans. on Antennas and Propagation, 2014. 